Supporting Information

\title{
Reconfigurable Self-Assembly and Kinetic Control of Multi-Programmed DNA-Coated Particles
}

\author{
Joon Suk $\mathrm{Oh}^{1}$, Gi-Ra Yi ${ }^{2, *}$, and David J. Pine ${ }^{1,3, *}$ \\ ${ }^{1}$ Center for Soft Matter Research, Department of Physics, New York University, New York, NY \\ 10003, USA \\ ${ }^{2}$ Department of Chemical Engineering, Sungkyunkwan University, Suwon, 16419 Republic of \\ Korea \\ ${ }^{3}$ Department of Chemical and Biomolecular Engineering, New York University, Brooklyn, NY. \\ 11201
}




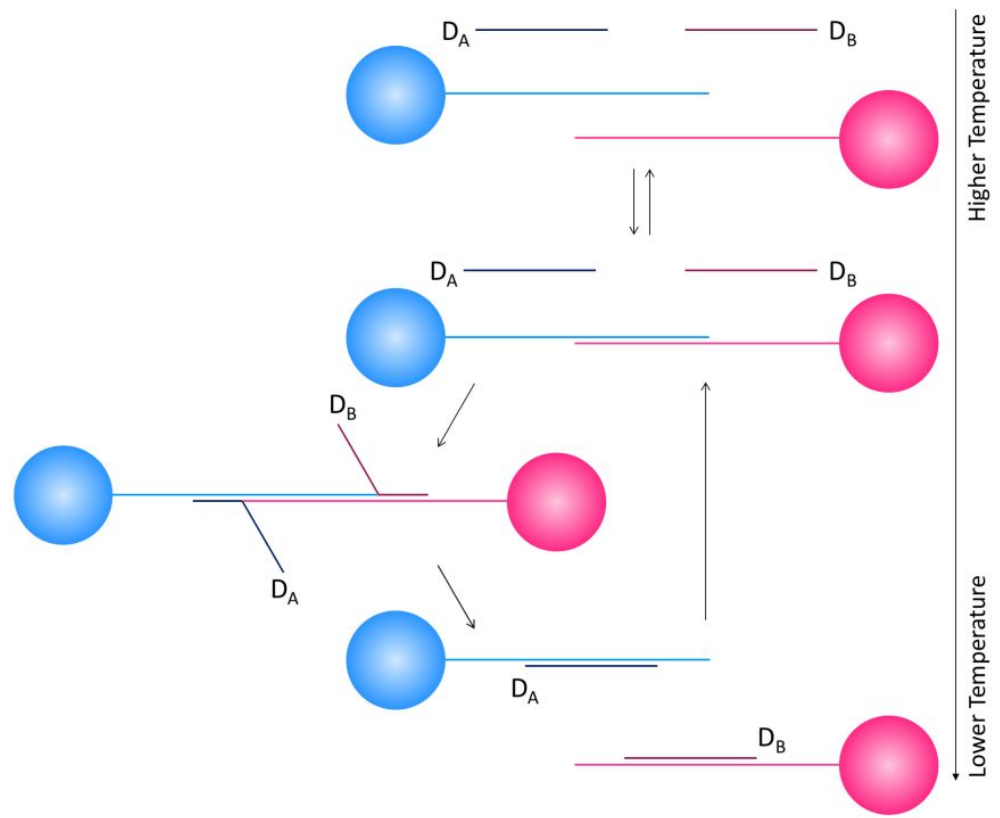

Figure S1. Association-dissociation of DNA-coated particles in the presence of displacement strands. 
(A)
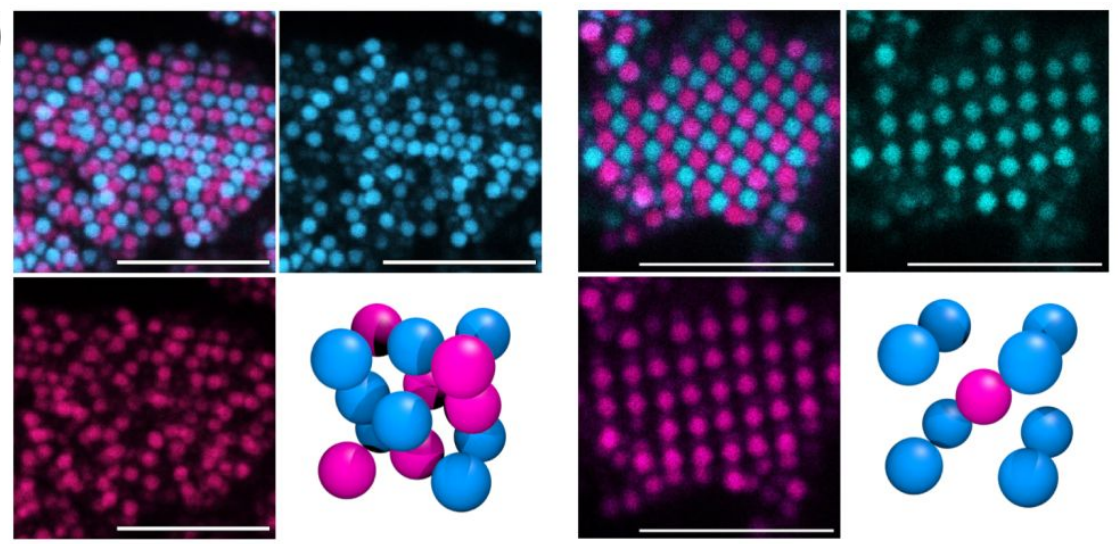

(B)
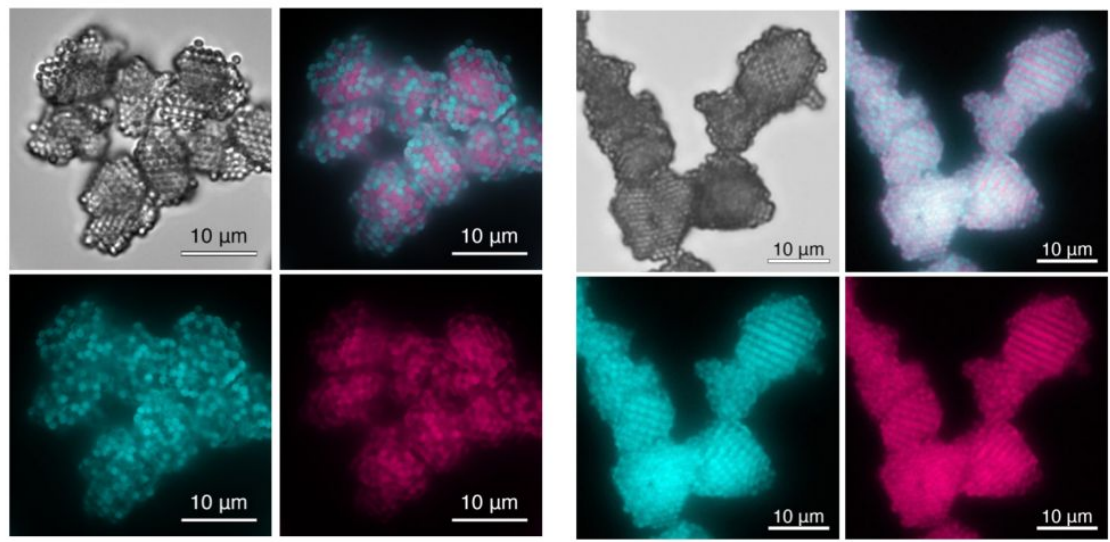

Figure S2. A, confocal microscope images of fcc (left) and $\mathrm{CsCl}$ (right) crystal structures. The fcc crystal structure is obtained at lower annealing temperature and the $\mathrm{CsCl}$ crystal structure at higher annealing temperature. B, bright-field and fluorescent images of fcc (left) and $\mathrm{CsCl}$ (right) crystal structures. The sample is recrystallized after more than dozens of thermal cycles. Scale bars 10 $\mu \mathrm{m}$. 


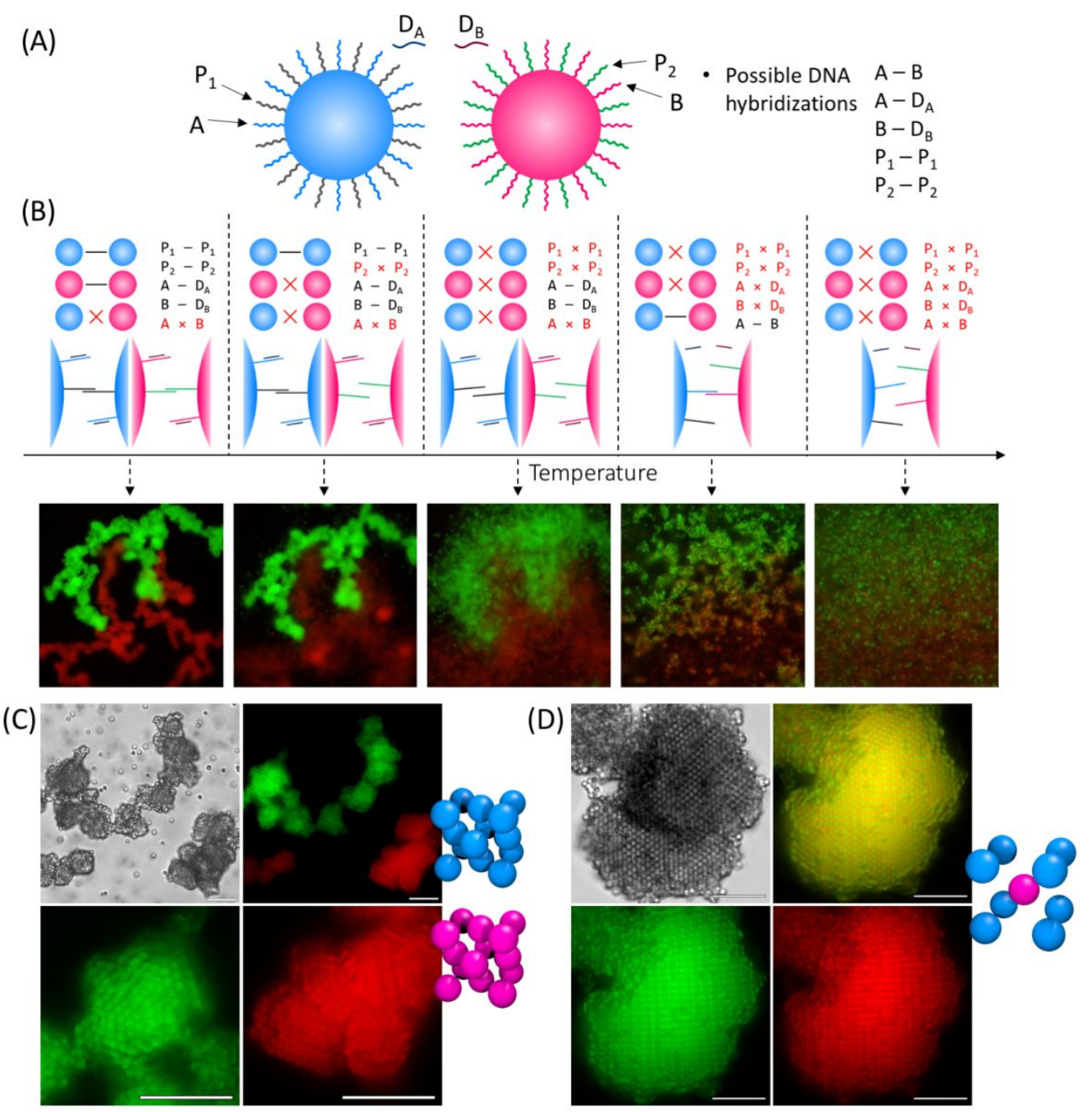

Figure S3. Phase transition and reconfigurable self-assembly of DNA-coated particles with the same sizes. A, two types of DNA-coated particles are prepared. Blue particles (green in the optical images) are coated with A DNA (complementary to B) and $\mathrm{P}_{1}$ DNA (self-complementary), and red particles are coated with $\mathrm{B}$ DNA and $\mathrm{P}_{2}$ DNA (self-complementary). $\mathrm{P}_{1}$ and $\mathrm{P}_{2}$ do not bind with each other. $\mathrm{D}_{\mathrm{A}}$ and $\mathrm{D}_{\mathrm{B}}$ are displacement strands for $\mathrm{A}$ and $\mathrm{B}$ strands, respectively. $\mathrm{B}$, illustration displays the specific interactions between DNA-coated particles, and the available DNA-DNA bonds as a function of temperature. Fluorescent images show corresponding states of the sample. $\mathbf{C}$, bright-field and fluorescent images show two separate fcc crystals and $\mathbf{D}, \mathrm{CsCl}$ crystal. Scale bars $10 \mu \mathrm{m}$.

The blue particles are coated with $\mathrm{A}$ and $\mathrm{P}_{1}$ DNA strands and the red particles are coated with $\mathrm{B}$ and $\mathrm{P}_{2}$ DNA strands (Figure $\mathrm{S} 3 \mathrm{~A}$ ). $\mathrm{P}_{1}$ and $\mathrm{P}_{2}$ DNA strands have self-complementary sticky ends, designed not to bind with each other. We used the same $A, B, D_{A}, D_{B}$ DNA strands mentioned 
above. The binding strength between the strands are following: A-B $>A-D_{A}, B-D_{B}>P_{1}-P_{1}>P_{2}-$ $\mathrm{P}_{2}$. Figure $\mathrm{S} 4 \mathrm{~B}$ shows the specific interactions between particles and the phase transition as a function of temperature. At the lowest temperatures, the A and B strands on the blue and red particles are deactivated by the displacement strands. Thus, blue particles bind with themselves through $\mathrm{P}_{1}$ strands and red particles bind through $\mathrm{P}_{2}$ strands. When the temperature increases, the red particles dissociate first since the binding strength of $\mathrm{P}_{2}$ strands is lower than that of $\mathrm{P}_{1}$. When the temperature increases further, blue particles dissociate as the $\mathrm{P}_{1}$ strands dehybridize. Further increase of the temperature breaks the $A-D_{A}$ and $B-D_{B}$ bonds resulting in the association of blue and red particles through complementary A-B strands. Fluorescent images in Figure S4B and video 3 clearly show the association-dissociation transitions as a function of temperature. Figure S4C shows the bright-field and fluorescent microscope images of two separate fcc crystals. We gradually increased the temperature to around $60^{\circ} \mathrm{C}$ and annealed. Figure S4D shows the brightfield and fluorescent images of $\mathrm{CsCl}$ crystals composed of blue and red particles. 


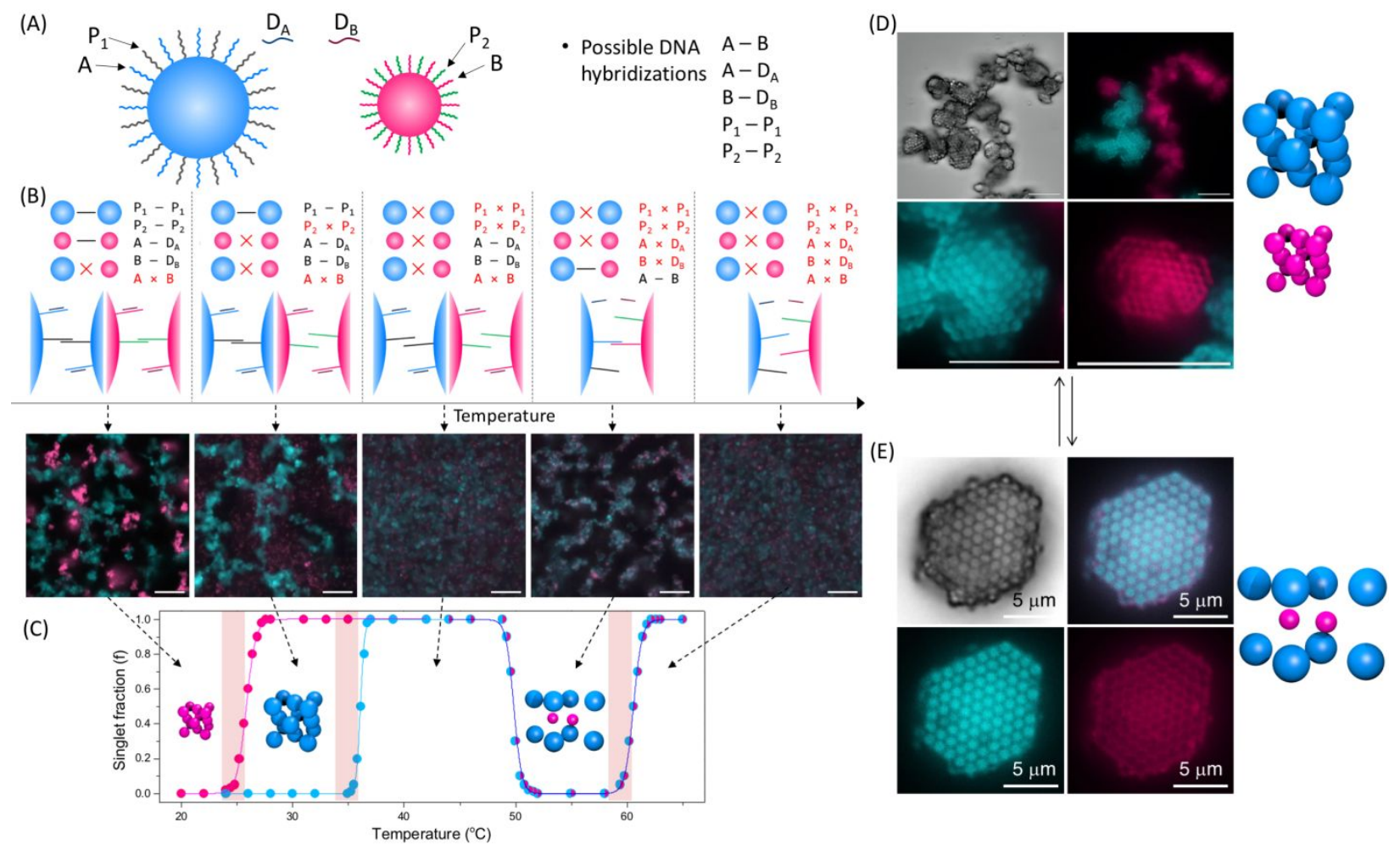

Figure S4. Phase transitions and reconfigurable self-assembly of DNA-coated particles of different sizes (1 and $0.6 \mu \mathrm{m}$ ). A, two types of DNA-coated particles are prepared. One is coated with A DNA (complementary to B) and $\mathrm{P}_{1}$ DNA (self-complementary), and the other one is coated with $\mathrm{B}$ DNA and $\mathrm{P}_{2}$ DNA (self-complementary). $\mathrm{P}_{1}$ and $\mathrm{P}_{2}$ do not bind with each other. $\mathrm{D}_{\mathrm{A}}$ and $\mathrm{D}_{\mathrm{B}}$ are displacement strands for $\mathrm{A}$ and $\mathrm{B}$ strands, respectively. $\mathbf{B}$, illustration displays the specific interactions between DNA-coated particles, and the available DNA-DNA bonds as a function of temperature. Fluorescent images show corresponding phases of the sample. $\mathbf{C}$, singlet fraction of the DNA-coated particles as a function of temperature. Red circle is for the singlet fraction of the smaller particles coated with $\mathrm{B}$ and $\mathrm{P}_{2}$ DNA. Blue circle is for the larger particles coated with $\mathrm{A}$ and $\mathrm{P}_{1}$ DNA. Two-colored circle is for both. D, bright-field and fluorescent images show two separate fcc crystals (111 planes), annealed around $25^{\circ} \mathrm{C}$ and $35^{\circ} \mathrm{C}$ and $\mathbf{E}, \mathrm{AlB}_{2}$ structures $(001$ plane), annealed around $60^{\circ} \mathrm{C}$. Scale bars $10 \mu \mathrm{m}$.

We prepared two types of DNA-coated particles with a different size ratio (1:0.6) to achieve different crystal structures since the assembling structure is also determined by the size ratio between building blocks (Figure S4). Blue particle is coated with A and $\mathrm{P}_{1}$ DNA strands, and red particle is coated with $\mathrm{B}$ and $\mathrm{P}_{2}$ DNA strands (Figure $\mathrm{S} 4 \mathrm{~A}$ ). $\mathrm{P}_{1}$ and $\mathrm{P}_{2}$ DNA strands have selfcomplementary sticky ends, designed not to bind with each other. We used the same $A, B, D_{A}, D_{B}$ 
DNA strands mentioned above. The binding strength between the strands are following: A-B $>$ A$D_{A}, B-D_{B}>P_{1}-P_{1}>P_{2}-P_{2}$. Figure $S 4 B$ shows the specific interactions between particles and the phase transition as a function of temperature. At the lowest temperature region, the $\mathrm{A}$ and $\mathrm{B}$ strands on blue and red particles are deactivated by the displacement strands. Thus, blue particles bind with themselves through $\mathrm{P}_{1}$ strands and red particles bind through $\mathrm{P}_{2}$ strands. When the temperature increases, the red particles dissociate first since the binding strength of $\mathrm{P}_{2}$ strands is lower than that of $\mathrm{P}_{1}$. When the temperature increases further, blue particles dissociate as the $\mathrm{P}_{1}$ strands unhybridize. Further increase in temperature breaks the $A-D_{A}$ and $B-D_{B}$ bonds, resulting in the association of blue and red particles through complementary strands (A-B). Fluorescent images in Figure S4B and video 4 clearly show the association-dissociation transitions as a function of temperature. Figure S4C displays the singlet fractions of blue, red, and both particles (a blue and red circle). Red particles first disassemble around $25^{\circ} \mathrm{C}$ followed by blue particles around $35^{\circ} \mathrm{C}$. No association of particles occurs between $36^{\circ} \mathrm{C}$ and $49^{\circ} \mathrm{C}$. Above $49^{\circ} \mathrm{C}$, the singlet fraction rapidly decreases to 0 as the particles assemble through $\mathrm{A}$ and $\mathrm{B}$ strands. Above $60^{\circ} \mathrm{C}$, all particles melt since all bonds break. We annealed the sample around $35^{\circ} \mathrm{C}$ first where blue particles crystalize into fcc structures. Then, we annealed it around $25^{\circ} \mathrm{C}$ annealing where red particles crystalize into another fcc structures. Figure S4D shows the bright-field and fluorescent microscope images of two separate fcc crystals. We gradually increased the temperature to around $60^{\circ} \mathrm{C}$ and annealed. Figure S4E shows the bright-field and fluorescent images of $\mathrm{AlB}_{2}$ crystals composed of blue and red particles. We confirmed that two separate fcc crystals can be reconfigured into $\mathrm{AlB}_{2}$ crystals at high temperature and vice versa. 

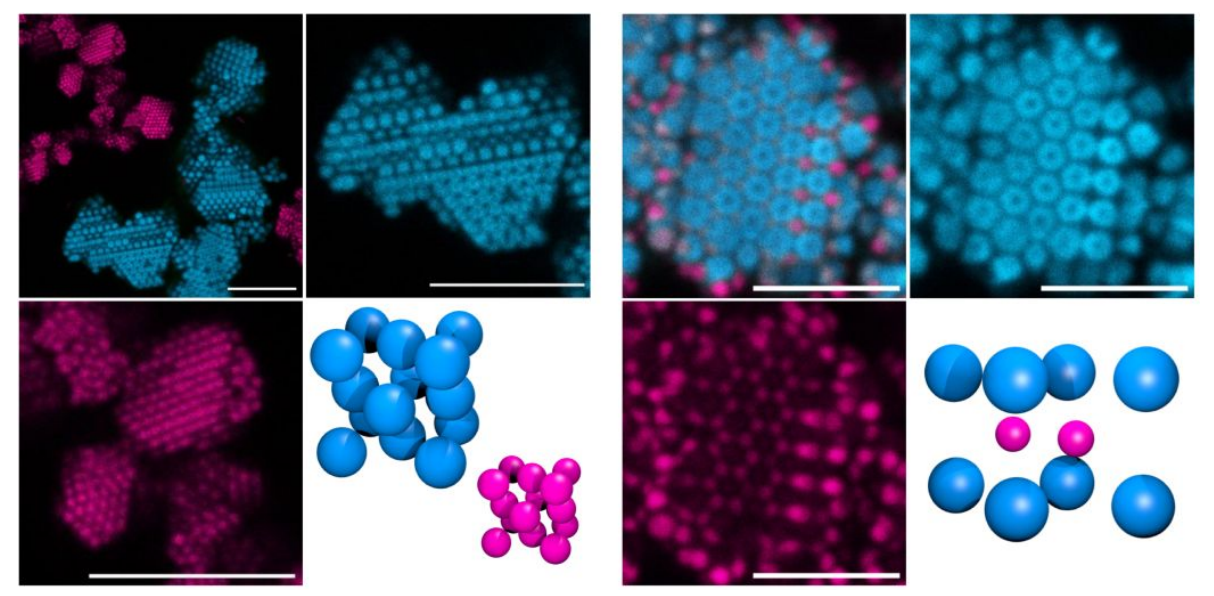

Figure S5. Confocal microscope images of two separate fcc (left) and $\mathrm{AlB}_{2}$ (right) crystal structures. Two separate fcc crystal structures are obtained at lower annealing temperatures and $\mathrm{AlB}_{2}$ crystal structure at higher annealing temperature. Scale bars $10 \mu \mathrm{m}$. 
(A)

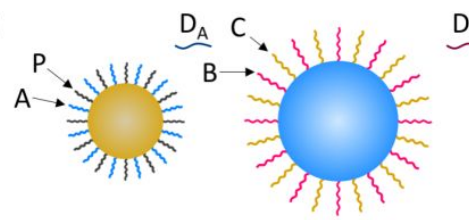

(B)

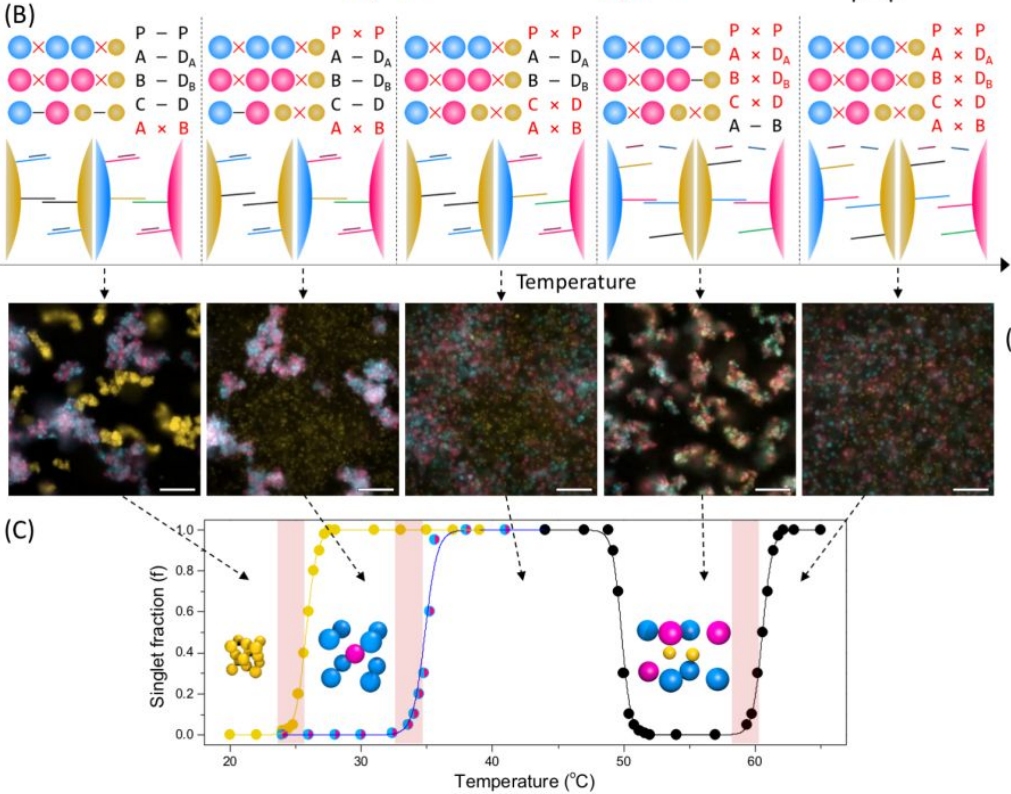

(D)
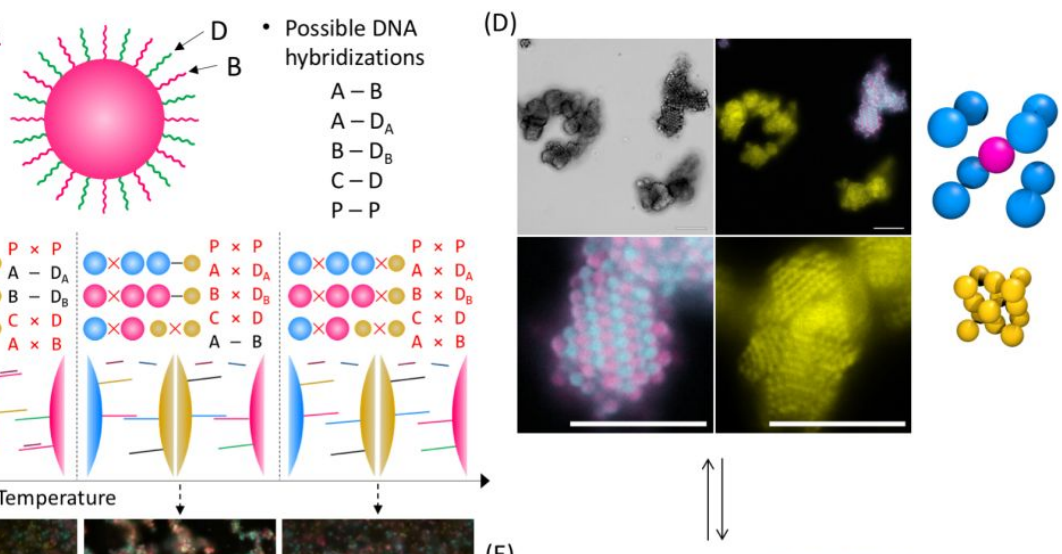

(E)

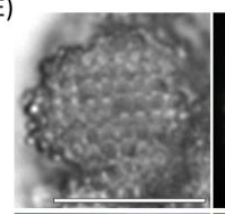

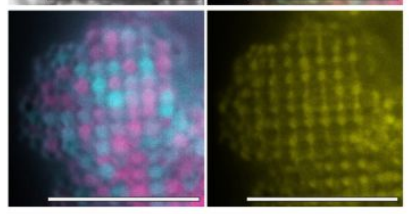

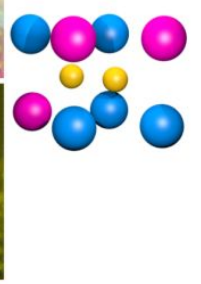

Figure S6. Phase transitions and reconfigurable self-assembly of DNA-coated particles of different sizes $(0.6,1$, and $1 \mu \mathrm{m})$. A, three types of DNA-coated particles are prepared. Yellow particle is coated with A DNA (complementary to B) and P DNA (self-complementary), blue particle is coated with B DNA and C DNA (complementary to D), and red particle is coated with B DNA and D DNA. $\mathrm{D}_{\mathrm{A}}$ and $\mathrm{D}_{\mathrm{B}}$ are displacement strands for $\mathrm{A}$ and $\mathrm{B}$ strands, respectively. B, illustration displays the specific interactions between DNA-coated particles, and the available DNA-DNA bonds as a function of temperature. Fluorescent images show corresponding phases of the sample. C, singlet fraction of the DNA-coated particles as a function of temperature. Yellow circle is for the singlet fraction of the small particles coated with A and P DNA. Two-colored circle is for the blue and pink particles. Black circle is for three particles. D, bright-field and fluorescent images show fec (111 plane) and $\mathrm{CsCl}$ crystals (110 plane), annealed around $25^{\circ} \mathrm{C}$ and $34^{\circ} \mathrm{C}$, and E, $\mathrm{AlB}_{2}$ crystals (100 plane), annealed around $60^{\circ} \mathrm{C}$. Scale bars $10 \mu \mathrm{m}$.

We explored a ternary system to demonstrate more complicated self-assembly of colloidal crystals.

Figure S6A illustrates three types of DNA-coated particles with different size ratio $(0.6: 1: 1)$.

Yellow particle is coated with A (complementary to B) and P DNA strands (self-complementary), and blue particle is coated with B and C DNA strands (complementary to D), and red particle is 
coated with $\mathrm{B}$ and $\mathrm{D}$ strands. $\mathrm{D}_{\mathrm{A}}$ and $\mathrm{D}_{\mathrm{B}}$ strands are used as displacement strands for $\mathrm{A}$ and $\mathrm{B}$ strands, respectively. The binding strength between the strands are designed in the following order: A-B $>A-D_{A}, B-D_{B}>C-D>$ P-P. Figure S6B shows the specific interactions and phase transition of DNA-coated particles as a function of temperature. At the lowest temperature, A and B strands are deactivated by the displacement strands. Thus, yellow particles bind with themselves through P strands (self-complementary), and blue particles can bind with red particles through C and D strands (complementary). When the temperature increases, yellow particles dissociate first as P-P binding breaks and then blue and red particles dissociate as C-D binding breaks. When the temperature increases further, $\mathrm{A}$ and $\mathrm{B}$ strands become active as the displacement strands liberate from A and B strands, leading to the association of yellow-blue particles and yellow-red particles through A-B binding. All particles dissociate above the melting temperature of A-B binding. The fluorescent images in Figure S6B and video 5 show the phase transitions of the particles as a function of temperature. Figure S6C shows the singlet fractions of the yellow particles, blue and red (a blue and red circle) particles, and all particles (black circle) as a function of temperature. It verifies that particles can transition sharply and reversibly. We annealed the sample at three different temperature regions. First, we annealed it around $34^{\circ} \mathrm{C}$, where blue and red particles crystalize into $\mathrm{CsCl}$ structures through $\mathrm{C}-\mathrm{D}$ binding. Then, we annealed around $25^{\circ} \mathrm{C}$, where yellow particles crystalize into fcc crystals through P-P binding. Figure S6D shows the bright-field and fluorescent microscope images of $\mathrm{CsCl}$ and fcc crystals. We gradually increased the temperature to around $60^{\circ} \mathrm{C}$ and annealed. We observed that fcc crystals melt first followed by $\mathrm{CsCl}$ crystals, and all particles rapidly assembled together and gradually turned into $\mathrm{AlB}_{2}$ crystal structures upon annealing (Figure $\mathrm{S} 6 \mathrm{E}$ ). Within the $\mathrm{AlB}_{2}$ structure, yellow particles bind with both 
red and blue particles since they are recognized as the same counterparts. As can be seen in the fluorescent image, red and blue particles are randomly distributed within the crystal lattice.
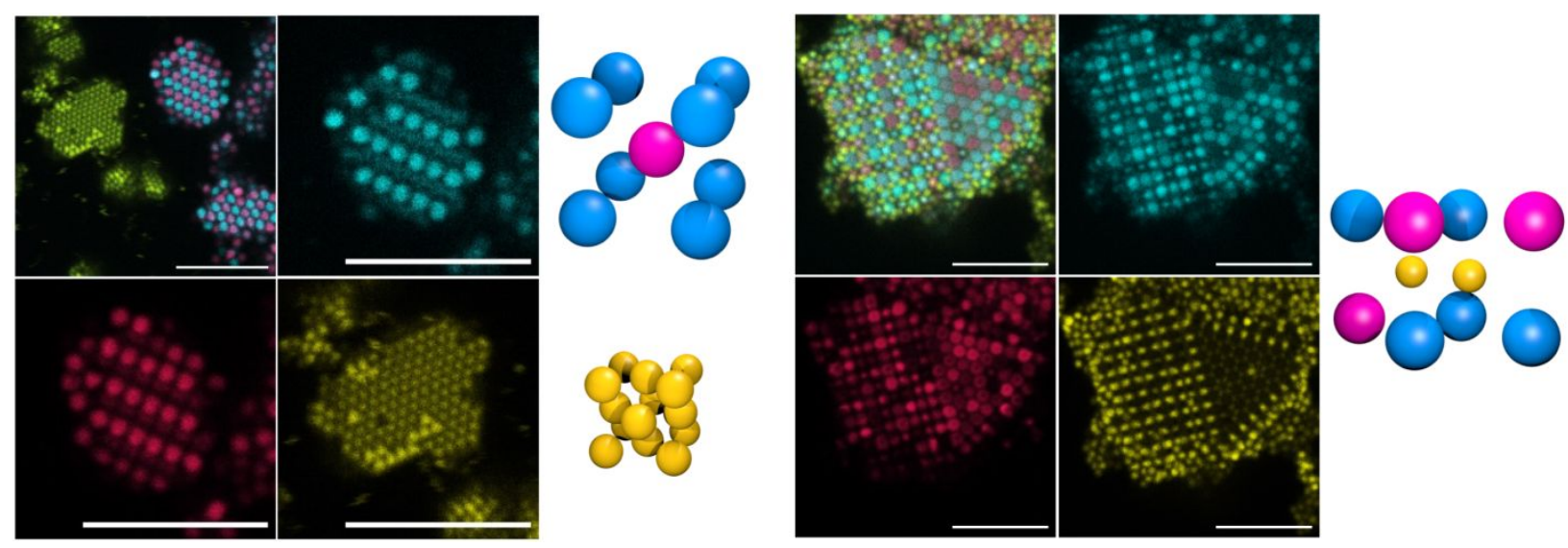

Figure S7. Confocal microscope images of fcc and $\mathrm{CsCl}$ (left), and $\mathrm{AlB}_{2}$ crystal structures. fcc and $\mathrm{CsCl}$ crystal structures are obtained at lower annealing temperatures and $\mathrm{AlB}_{2}$ crystal structure at higher annealing temperature. Scale bars $10 \mu \mathrm{m}$.

Video 1. Phase transition of DNA-coated PS particles of the same size (P-P and A-B).

Video 2. Crystal transformation from $\mathrm{CsCl}$ to fcc structures.

Video 3. Phase transition of DNA-coated PS particles of the same size $\left(\mathrm{P}_{1}-\mathrm{P}_{1}, \mathrm{P}_{2}-\mathrm{P}_{2}\right.$, and A-B).

Video 4. Phase transition of DNA-coated PS particles of different sizes (1 and $0.6 \mu \mathrm{m})$.

Video 5. Phase transition of DNA-coated PS particles of different sizes $(0.6,1$, and $1 \mu \mathrm{m})$. 